\title{
Strategies to Prevent Dendritic Cell-driven Infection Across the Mucosa
} Melissa Pope ${ }^{* \ddagger}$

Address: Center for Biomedical Research, Population Council, USA

Email: Melissa Pope* - mpope@popcouncil.org

* Corresponding author $\ddagger$ Presenting author

from 2005 International Meeting of The Institute of Human Virology Baltimore, USA, 29 August - 2 September 2005

Published: 8 December 2005

Retrovirology 2005, 2(Suppl I):S84 doi:I0.II86/I742-4690-2-SI-S84

Early events leading to HIV infection across the mucosa likely involve HIV capture by a wide variety of molecules on the surface of epithelial cells and leukocytes followed by infection of permissive target cells within the tissues. In studying this biology, we are focusing on the contribution of dendritic cells (DCs) and T cells to these events and exploring effective ways to block these complex events in vitro and in vivo. Earlier work confirmed that there are at least two phases of DC-driven transmission of virus to $\mathrm{T}$ cells - one involves virus captured by (but not infecting) DCs that is handed directly over to the T cells and the other involves DC infection and the transmission of newly synthesized viruses. Virus captured by DCs is transmitted to CD4 $\mathrm{T}$ cells moving rapidly across the synapse naturally created between DCs and T cells. Inclusion of the fusion inhibitor T1249 reduces the amount of virus movement to the T cells, while increasing the amount of virus accumulating in the DCs. The immunologic and virologic consequences of this are under investigation. These data highlight how only blocking certain pathways of virus-DC interactions are suboptimal in preventing DCdriven HIV spread. As a result, additional studies are being performed to test the ability of more broad-acting carrageenan-based formulations for their ability to impede the complex virus-cell interplay needed to facilitate transmission. Carrageenan-based microbicides are promising due to their wide rage of activity against HIV/SIV and other sexually transmitted pathogens. Carrageenans impair virus capture by DCs in vitro and block infection of permissive DC-T cell mixtures. Recent in vivo data revealed that macaques were protected against vaginal SHIV challenge by carrageenan-based microbicides. These data are encouraging for future application of carrageenan-based formulations in preventing HIV spread. 